\title{
Professor no comando: curso prático de desenvolvimento de chatbots para docentes
}

\author{
Francielli Freitas Moro - PPGIE/UFRGS - franf_m@hotmail.com \\ Rosa Maria Vicari - PPGIE/UFRGS - rosa@inf.ufrgs.br \\ Raquel Fiori - PPGQVS/UFRGS - raquelfiori2109@gmail.com \\ Liane Margarida Rockenbach Tarouco - PPGIE/UFRGS - liane@penta.ufrgs.br
}

Resumo. Permitir ao professor autonomia sobre a construção de sistemas para disponibilizar conteúdo e apoio aos alunos, é muito importante para aprimorar processos educacionais. Para que isto ocorra, é necessário oferecer capacitações que atendam os professores de maneira prática e objetiva. Este artigo descreve a criação, aplicação e avaliação de um curso introdutório de desenvolvimento de chatbots, utilizando o serviço Watson Assistant da IBM Cloud. O curso foi aplicado com um grupo de professores voluntários, através das plataformas Zoom e Google Classroom, utilizando o conceito de Sala de Aula Invertida. Para obtenção de resultados, foi disponibilizado um questionário online além das observações e discussões realizadas nas aulas. O questionário apresentou além da obtenção nova de conhecimento sobre chatbots e seu desenvolvimento prático, motivação e engajamento para aplicação futura das habilidades obtidas como apoio ao conteúdo disponibilizado em sala ou no ambiente e-learning.

Palavras-chave: chatbots; sala de aula invertida; desenvolvimento de agentes conversacionais; curso para professores; Watson.

\section{Teacher in Charge: practical course on developing chatbots for teachers}

\begin{abstract}
Allowing the teacher autonomy over the construction of systems to provide content and support to students is very important for the improvement of educational processes. For this to occur, it is necessary to offer training that serves teachers in a practical and objective way. This paper describes the creation, application and evaluation of an introductory chatbots development course, using the IBM Cloud Watson Assistant service. The course was applied with a group of volunteer teachers, through the Zoom and Google Classroom platforms, using the concept of flipped classroom. To obtain results, an online questionnaire was available in addition to observations and discussions held in class. The questionnaire presented in addition to obtaining new knowledge about chatbots and their practical development, motivation and engagement for the future application of skills obtained in support of content in class or in the e-learning environment.
\end{abstract}

Keywords: chatbots; development of conversational agents; course with teachers; Watson.

\section{Introdução}

Com o avanço e perspectivas futuras de Tecnologias da Informação e Comunicação (TIC's), além da consequente pandemia de Sars-cov-19, vivencia-se um período de adaptações e oportunidades para inserção de ferramentas inteligentes dentro do contexto 
educacional que apoiem o estudante no ambiente e-learning e os entreguem feedbacks imediatos. No sentido de aplicação, Beira e Nakamoto (2016) defendem a necessidade de uma capacitação docente intensiva tecnológica, em que professores e desenvolvedores trabalhem juntos para a construção de conhecimento sobre as ferramentas a serem utilizadas em conjunto do seu domínio, a fim de criar práticas inovadoras.

A formação continuada de professores com o uso da sala de aula invertida e ensino híbrido, foi aspectos abordados por Morais e Souza (2020) e Dos Santos e Tezani (2018). Os autores ponderam que, apesar de todas as dificuldades e empecilhos vivenciados pelos professores no cenário nacional, é possível que a escola e a universidade articulem em suas discussões, experiências práticas na formação. As tecnologias digitais têm mudado a maneira de ver o mundo e conviver no espaço social, sempre com a necessidade de otimizar o tempo e agilizar a vida.

Um chatbot, também denominado agente conversacional, é um agente que atua de forma inteligente dentro de um sistema e-learning, por exemplo, interagindo com um aluno ou professor, de maneira próxima ao natural, para oferecer conteúdo adaptável, responder dúvidas, recomendar conteúdos, entre outras funções (Ndukwe, Daniel e Amadi, 2019). Para o desenvolvimento de um chatbot, atualmente, existem serviços de Application Programming Interface (API) e sistemas desenvolvidos por empresas, que possuem uma interface simples e objetiva. Esses serviços facilitam para que uma pessoa, sem conhecimentos prévios de programação, consiga desenvolver um chatbot de forma usual e disponibilize aos usuários. Os serviços mais conhecidos são o Watson Assistant da IBM e o Dialogflow da Google (Barros e Guerreiro, 2019). A possibilidade do professor poder criar uma base de conhecimento de um sistema a hora que ele quiser e disponibilizá-lo ao seu aluno para o apoiá-lo com feedback's imediatos sobre o conteúdo estudado, é um passo muito importante em sistemas educacionais. Para que isto ocorra é necessário oferecer capacitação adequada, em cursos que os ensinem a aproveitar da melhor maneira possível os recursos disponíveis de acordo com sua necessidade.

Este artigo tem o objetivo de apresentar a criação, apresentação e avaliação de um curso introdutório de desenvolvimento de chatbot através do serviço Watson Assistant em seu plano lite, presente no catálogo IBM cloud. Os participantes do curso foram professores voluntários, que atuam em diversos níveis de ensino público e privado nas áreas de Ciências, Tecnologias, Humanidades, Engenharias e Matemática (STHEM) em instituições no Brasil. A finalidade do curso era possibilitar aos professores conteúdos e experiências de "faça você mesmo" para o desenvolvimento dos seus próprios agentes. Para isto, utilizou-se o modelo de sala de aula invertida a partir da plataforma Zoom, onde aconteceram as aulas síncronas; e a plataforma Google Classroom, onde foram disponibilizados os materiais prévios e interações pós-aulas.

Não foram encontradas nas buscas realizadas nas bases Scopus, Google Scholar e na Revista Renote, em outubro/novembro de 2020, utilizando as palavras-chave: "curso", "sala de aula invertida", "chatbots" e "Watson" (considerando suas correspondentes em inglês), nenhuma pesquisa que satisfaça o objetivo apresentado na introdução deste artigo.

O artigo está dividido da seguinte forma, a partir desta seção introdutória. Na seção 2, são apresentados os conceitos e atribuições relacionadas a chatbots na educação a partir de pesquisas e ao funcionamento do Watson Assistant. Na seção 3, é apresentada 
a metodologia utilizada para o desenvolvimento deste projeto. Na seção 4, apresenta-se aplicação do questionário e discussão sobre os resultados obtidos com os participantes do curso, e na seção 5, são realizadas as conclusões.

\section{Chatbots na educação}

Para compor esta seção, foi realizada uma busca exploratória a partir da pesquisa de uma das autoras, e são apresentados alguns trabalhos que se destacaram por suas diferenças e relevâncias, e serviram de base na criação do curso.

O trabalho de Huang, Hew e Gonda (2019), apresenta o desenvolvimento e avaliação de três chatbots para apoiar o aprendizado, em um curso que utiliza metodologia de sala de aula invertida. Como resultado das interações dos alunos com o chatbot, obteve-se a redução da sensação de isolamento do aprendizado e conhecimentos sobre conceitos utilizados no curso. Porém, notou-se a necessidade de uma melhor construção de uma base de conhecimento para respostas mais assertivas e dinâmicas

A professora Elektra, apresentada por (Leonhardt et al, 2003), foi desenvolvida em 2002 para auxiliar o aprendizado de estudantes na área de física, na sua preparação para o vestibular, ganhando, em 2003, uma extensão para ensino de redes de computadores e informática. Elektra tem como base de desenvolvimento o chatbot ALICE conhecido por sua introdução a linguagem AIML.

O trabalho de Moro (2019) apresenta um chatbot para um Sistema Tutor Inteligente (STI) MAZK que tem como objetivo responder as dúvidas de professores sobre o STI e suas metodologias. O agente é baseado na estruturação de diversos sistemas criados por empresas como Microsoft, Facebook e IBM, embora, sua base de conhecimento seja reduzida. Os resultados mostram uma forte aceitação do uso da ferramenta por parte dos docentes.

Yang e Evans (2019) apresentam três chatbots desenvolvidos para apoiar o estudante em uma universidade. O primeiro auxilia na entrega de um jogo de simulação de um curso de mestrado; o segundo no treinamento e uso de um sistema educacional; e o terceiro no processamento de solicitações de ajuda sobre a universidade.

A maior diferença entre os três exemplos apresentados, além do objetivo e aplicação, é o desenvolvimento. Enquanto Elektra utiliza a linguagem baseada em regras AIML em sua base, o agente do STI MAZK, utiliza linguagem de programação com técnicas de Linguagem Natural de Máquina e Redes Neurais Artificiais. Os chatbots de Yang e Evans (2019), por sua vez, foram construídos via serviço de API do Watson, denominado Watson Assistant.

Kuyven et al (2018) apresentam uma revisão sistemática sobre agentes conversacionais, aplicados na educação, em que destacam o fato de todas as pesquisas utilizarem a técnica de AIML em sua construção. A principal razão para isto, está na facilidade de acesso e disponibilização, mas considera o uso de outras técnicas, como uma possível aplicação utilizando o Watson Assistant.

\subsection{IBM Watson}

O Watson Assistant, segundo sua documentação (IBM, 2020) trabalha a partir de instâncias, em que são necessárias para criação de um diálogo simples. Os principais conceitos que o usuário precisa conhecer são:

1)Intenção: objetivos que o usuário busca com a interação com o bot; 
2)Entidade: termos/objetos que fornecem o contexto para uma intenção;

3)Diálogo: fluxo da conversa.

Não são necessários os conhecimentos prévios de programação para construção de chatbots simples de interação utilizando o serviço IBM. Em geral são requeridos apenas conhecimentos sobre o sistema e como ele atua utilizando os tópicos acima. A interface do Watson é bastante amigável e oferece também um compilador visual bem próximo a um chat como o do Whatsapp. Este fator, permite ao usuário desenvolvedor um entendimento maior sobre o produto à medida que vai atribuindo novos conhecimentos à base.

Há também a possibilidade de integração do serviço com websites e plataformas de chats como o Messenger, Telegram e WeChat. Para realizar a integração são necessários alguns conhecimentos mais detalhados, porém, o Watson oferece, aos usuários, um link dentro do próprio serviço com uma interface de chat amigável, que o professor pode disponibilizar ao aluno para utilização do bot criado.

É importante frisar que a versão gratuita da ferramenta possuí algumas limitações quanto a número de requisições de acesso e quantidade de instâncias que podem ser criadas. Mesmo este número sendo alto, existem outras opções de planos, caso haja esgotamento. Apesar de não requerer conhecimentos específicos em linguagens de programação, o uso do serviço IBM Watson pode ser complexo para professores menos familiarizados com sistemas tecnológicos, necessitando assim capacitação e atendimento adequado.

\section{Metodologia}

Foi realizado um curso introdutório com o total de 12 horas/aulas distribuídas em 2 aulas síncronas de 2 horas, e o restante em aulas assíncronas realizadas ao longo de 3 semanas. O curso foi aplicado com professores voluntários, de diversas instituições públicas e privadas, que atuam na área de STHEM no Brasil, principalmente na região sul e sudeste. Os professores são colaboradores no grupo STHEM Brasil (https://www.sthembrasil.com/), responsável por contatá-los. Este curso foi idealizado considerando as dificuldades relacionadas a autonomia dos professores a produção de sistemas e objetos de aprendizagem, necessitando muitas vezes de mão de obra custosa e deslocamento. Estas dificuldades acabam criando resistências e desistências na utilização de inovações tecnológicas. $O$ curso foi ministrado por 3 pesquisadoras mulheres da UFRGS, sendo: 1 doutora do Instituto de Informática que atua na área de Inteligência Artificial (IA); 1 doutoranda em Informática na Educação que atua no desenvolvimento de chatbots com IA, especialista na ferramenta Watson Assistant; e 1 doutoranda em educação em ciências e professora na área de STHEM. Foi utilizado o modelo de sala de aula invertida (adaptado, devido a pandemia de Sars-cov-2), e foi realizado em duas etapas principais, divididas em módulos:

Etapa 1: Aulas assíncronas distribuídas em 8h/aula, que foram alocadas em uma sala virtual no Google Classroom. O conteúdo destas aulas é composto por materiais teóricos e explicativos sobre chatbots, bem como uma introdução ao Watson Assistant.

Etapa 2: Aulas Síncronas distribuídas em 4h/aula, realizadas em uma sala virtual na plataforma ZOOM, para aplicação dos conhecimentos obtidos, e discussão sobre dúvidas. 
O quadro 1, apresenta detalhadamente todos os módulos e atribuições de cada etapa e desenvolvimento do curso.

Quadro 1 - Módulos e aplicação das etapas 1 e 2.

\begin{tabular}{|c|c|}
\hline \multirow[b]{2}{*}{ Módulo } & \multirow[b]{2}{*}{ Aplicação } \\
\hline & \\
\hline $\begin{array}{l}\text { Módulo 1: Apresentação da equipe e } \\
\text { objetivos do curso. }\end{array}$ & $\begin{array}{l}\text { Apresentação Textual utilizando } \\
\text { Plataforma Google Classroom }\end{array}$ \\
\hline $\begin{array}{l}\text { Módulo 2: Apresentação dos conceitos e } \\
\text { funcionamento de maneira teórica sobre um } \\
\text { chatbot, bem como suas aplicações. Aula } \\
\text { teórica. }\end{array}$ & $\begin{array}{l}\text { Apresentação de artigos, slides, textos e } \\
\text { vídeos sobre o tema, alocados na Plataforma } \\
\text { Google Classroom (assíncrono). }\end{array}$ \\
\hline $\begin{array}{l}\text { Módulo 3: Apresentações iniciais do IBM } \\
\text { Watson, e introdução prática na ferramenta. }\end{array}$ & $\begin{array}{l}\text { Apresentação de vídeo e slide, alocados na } \\
\text { Plataforma Google Classroom(assíncrono). }\end{array}$ \\
\hline $\begin{array}{l}\text { Módulo 4: Discussões sobre o material } \\
\text { apresentado previamente, ensino passo-a- } \\
\text { passo de como utilizar a ferramenta e atribuir } \\
\text { conceitos apresentados. }\end{array}$ & $\begin{array}{l}\text { Apresentação por videoconferência através } \\
\text { da plataforma Zoom, e o IBM Watson } \\
\text { Assistant para desenvolvimento (síncrono). }\end{array}$ \\
\hline $\begin{array}{l}\text { Módulo 5: Discussões, no fórum, sobre o } \\
\text { material apresentado até o momento e } \\
\text { dúvidas sobre a tarefa atribuída. }\end{array}$ & $\begin{array}{l}\text { Fórum de Discussão criado na Plataforma } \\
\text { Google Classroom (assíncrono). }\end{array}$ \\
\hline $\begin{array}{l}\text { Módulo 6: Revisão dos conceitos e } \\
\text { aplicações prévias, estudo de novos } \\
\text { materiais, criação inicial do chat adaptável a } \\
\text { cada conteúdo. }\end{array}$ & $\begin{array}{l}\text { Conteúdo disponibilizado na } \\
\begin{array}{l}\text { Google Classroom e busca em outros } \\
\text { sites/documentos de forma individual. } \\
\text { (assíncrono). }\end{array}\end{array}$ \\
\hline $\begin{array}{l}\text { Módulo 7: Continuação da explicação passo- } \\
\text { a-passo da ferramenta atribuindo os conceitos } \\
\text { apresentados durante o curso, a partir da } \\
\text { tarefa atribuída. }\end{array}$ & $\begin{array}{l}\text { Apresentação por videoconferência através } \\
\text { da plataforma Zoom, e o IBM Watson } \\
\text { Assistant para desenvolvimento (síncrono) }\end{array}$ \\
\hline $\begin{array}{l}\text { Módulo 8: Finalização do chatbot criados } \\
\text { pelos professores, respostas ao questionário } \\
\text { de avaliação; discussões, dúvidas e sugestões } \\
\text { de materiais de forma colaborativa no } \\
\text { Classroom. }\end{array}$ & $\begin{array}{l}\text { Aplicação do conteúdo estudado utilizando o } \\
\text { IBM Watson; utilização do Google } \\
\text { Classroom para o conteúdo complementar e } \\
\text { acompanhamento de dúvidas; e respostas ao } \\
\text { questionário de avaliação na plataforma } \\
\text { Google Forms. (assíncrono). }\end{array}$ \\
\hline
\end{tabular}

Para avaliar a aplicação do curso e dos conhecimentos obtidos, além da observação e interação durante as aulas síncronas, foi construído um questionário com teor quantitativo e qualitativo, composto por 11 perguntas. As perguntas avaliaram, além da obtenção de novos conhecimentos sobre chatbots e seu desenvolvimento prático, motivação e engajamento para aplicação futura das habilidades obtidas como apoio ao conteúdo disponibilizado em sala ou no ambiente e-learning. O questionário foi desenvolvido na plataforma Google Forms e foi disponibilizado ao fim do curso, no ambiente e-learning, para que os professores pudessem avaliar e sugerir melhorias para próximas edições desse curso. De todos os professores que participaram do curso, 15 responderam ao questionário online. 


\section{Aplicação e Resultados}

Como apontado no Quadro 1, da Seção 3, todos os materiais foram fornecidos de forma prévia na plataforma Google Classroom, que foram divididos em 3 tópicos principais; Apresentações, Preparação para o hands-on (alocação de textos, slides, vídeos) e Avaliação. O Mural serviu para a atribuição de fóruns de discussões e links complementares. Alguns alunos se mostraram motivados e engajados em sua utilização. A Figura 1, mostra a organização do curso no Classroom.

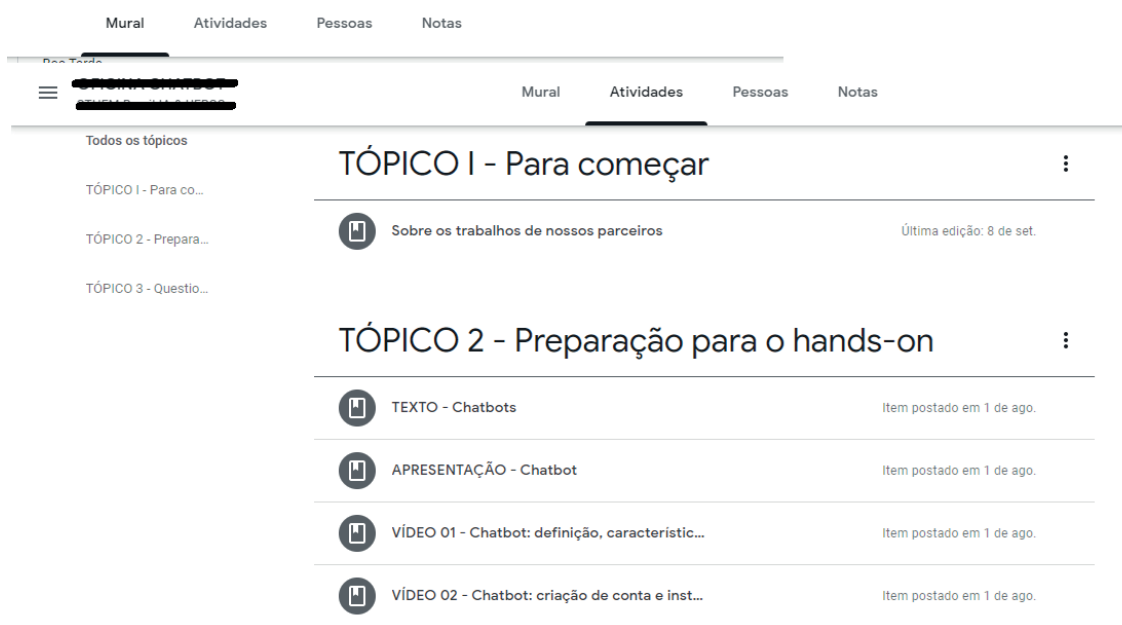

Figura 1 - Organização do curso no ambiente e-learning

Uma das alunas criou dois tutoriais a partir das aulas apresentadas e do conteúdo complementar, para disponibilizar para seus alunos da disciplina de Automação de Escritório e Secretariado. O tutorial, desenvolvido pela professora, foi alocado no mural e mostrou empenho e aceitação por parte da mesma em aplicar aquilo que era ensinado não só para criar seu próprio agente, mas também de repassar o que aprendeu a seus alunos.

As aulas realizadas no Zoom contaram com a duração de $2 \mathrm{~h}$ cada, e participação ativa dos alunos, que desenvolviam o exemplo junto com a tutora, ou seja, de forma síncrona. Notou-se que quase metade dos professores não haviam acessado todo o material prévio do curso ou não haviam se inscrito na plataforma IBM Cloud, o que demandou uma boa parte de tempo da primeira aula, para os nivelar principalmente em relação ao cadastro e criação de instância no serviço Watson Assistant. Estes passos foram previamente explicados em vídeos e disponibilizados aos participantes. A não visualização atrasou um pouco o planejamento, ficando boa parte de criação e ajuste de entidades para a segunda aula. Por esse motivo, observa-se a necessidade de frisar aos professores o acesso aos vídeos antes da aula pois a metodologia empregada pressupunha esta etapa prévia. No segundo dia de alunas síncronas o conteúdo prático foi finalizado.

O questionário de avaliação disponibilizado no ambiente e-learning possibilitou verificar o potencial do curso para ensino de uma ferramenta útil de forma prática, para professores. Verificou-se a partir dele que os professores respondentes tinham entre $30 \mathrm{a}$ 59 anos, a maioria lecionando em cursos de graduação de entidades públicas e privadas, no Brasil. principalmente nas áreas de Engenharia, Matemática e Tecnologia. Cinco (5) deles apontaram que não gostam de interagir com chatbots como usuários. Quando perguntados, se suas experiencias de interações com agentes foram efetivas, 7 
responderam que sim, 4 que não e os outros responderam que algumas vezes foram, mas outras não. Com este quadro inicial, podemos perceber que embora não sejam nativos digitais a maioria conhece ou já interagiu com chatbots e que entendem seu funcionamento e aplicabilidade. Um exemplo de interação anterior citada pelos professores foi a utilização de chatbots de instituições de ensino para solucionar dúvidas sobre serviços e informações sobre o ambiente.

Quando perguntados sobre qual o aprendizado obtido com o curso, a partir de uma questão múltipla escolha, os pontos mais destacados foram: Entender conceitos sobre chatbot, conhecer a ferramenta Watson e Desenvolver um chatbot de fato. Nenhum professor disse que não aprendeu algo novo com o curso. Ainda, 4 relacionaram o entendimento de conceitos sobre Inteligência Artificial como mostrado no gráfico da Figura 2.

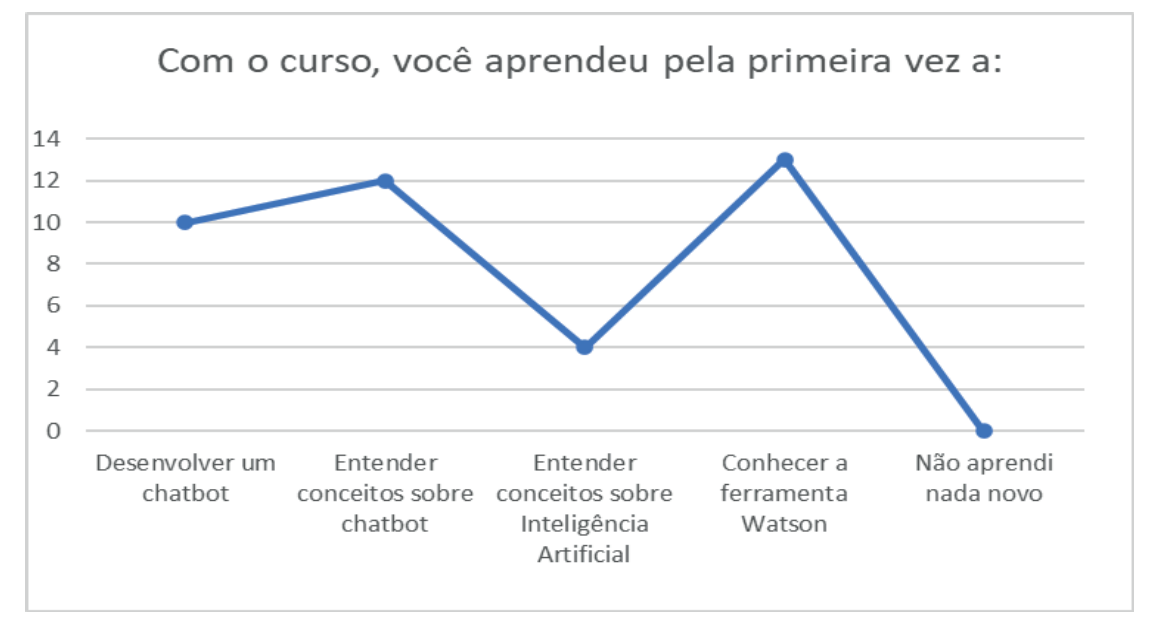

Figura 2 - Análise de aprendizado obtido no curso

A respeito dos entendimentos sobre chatbots antes e depois do curso, foi solicitado que os professores o classificassem no intervalo de 0 até 5 , onde pouco (corresponde o valor mínimo de 0) até muito (corresponde o valor máximo de 5). Os Gráficos da Figura 3 , corresponde o nível de conhecimento dos professores, sobre o conteúdo, antes (3.a) e depois (3.b) do curso.

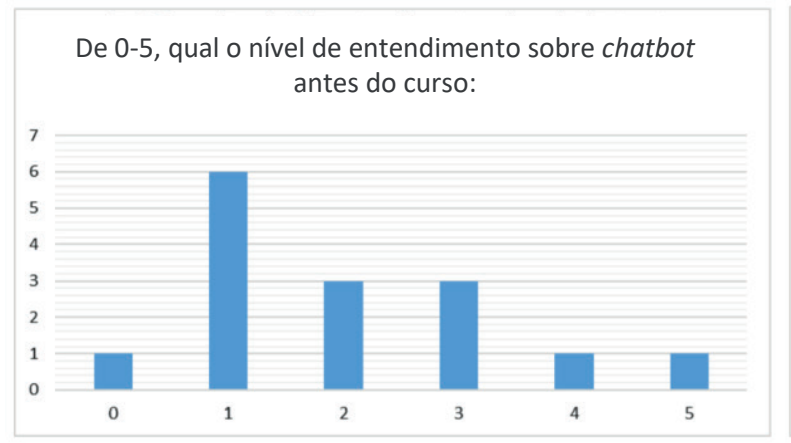

(a)
De 0-5, qual o nível de entendimento sobre chatbot depois do curso:

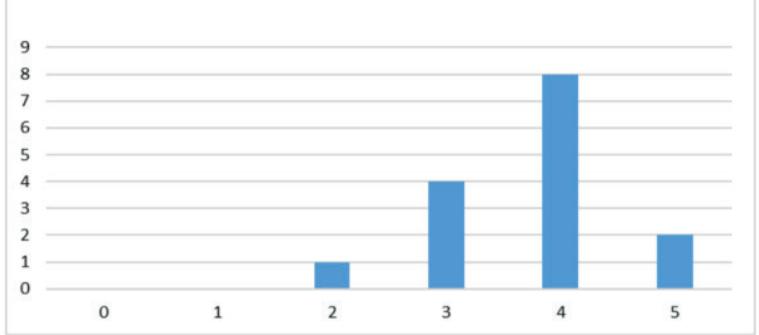

(b)

Figura 3 (a,b) - Classificação de conhecimento sobre conceitos e desenvolvimento de chatbot anterior e posterior ao curso

Neste caso, como mostra a Figura 3.a, para ambos os extremos [0 e 5] apenas 1 professor classificou como pouco ou muito, mas podemos notar que a maioria classificou seu conhecimento prévio como 1 ou 2 , o que significa dizer que estes 
consideram não saber muito sobre chatbots antes do início do curso. Relacionando-se esta informação com o fato de que a maioria já havia interagido com chatbots anteriormente e com as discussões obtidas durante as aulas síncronas, verificou-se que a maioria havia apenas utilizado o chatbot como usuário. Ou seja, grande parte dos participantes desconhecia os conceitos e aplicações destes, nem sobre seu desenvolvimento.

O gráfico da Figura 3.b solicita aos professores para classificarem o nível de entendimento sobre chatbots depois do curso. E, a partir dele nota-se que os níveis 0 e 1, mais baixos na escala não foram selecionados, e apenas 1 professor selecionou $o$ nível 2.

A maioria dos respondentes selecionou os níveis 4,5 e 6 de classificação sobre entendimento adquirido após o curso (com a maior concentração no nível 4), o que possibilita verificar que a maior parte dos professores aprenderam consideravelmente com a metodologia utilizada, ferramenta aplicada, conceitos teóricos e atribuições práticas.

Os professores ainda responderam duas questões discursivas a respeito da utilização do chatbot em desenvolvimento para aplicação em suas disciplinas. Neste contexto, quando perguntados qual propósito eles buscavam de utilização em suas atividades, a maioria respondeu: buscar a disponibilização de uma ferramenta adaptável e interativa para seus alunos e oferecer tutoria e feedback imediato. Neste sentido, foi possível verificar que os participantes entenderam os conceitos de aplicação explicados no curso, e que seus objetivos estão alinhados com o propósito do curso. Houve também respostas como auxiliar sua instituição na criação de agentes que respondam sobre serviços disponibilizados, mostrando a busca por aplicabilidade além da sala de aula.

A segunda pergunta discursiva, está relacionada com como os professores utilizariam esse chatbot com seus alunos de acordo com seu objetivo. As respostas foram: "Como ferramenta de apoio em aulas invertidas e revisões de conteúdo", "Na automatização de processos de trabalho, como conteúdo para o desenvolvimento da literacia digital e pensamento computacional de meus estudantes", entre outras.

Algumas sugestões visando o aprimoramento do curso também foram recebidas, uma delas está relacionada ao tempo atribuído para as aulas, em que o atraso inicial prejudicou a distribuição do conteúdo, resultando na necessidade de mais horas aulas.

Alguns professores já tinham uma noção maior de ambientes computacionais e estruturas, tendo um rendimento melhor no curso. Isso mostrou a necessidade de um segundo módulo avançado visando solucionar as dúvidas mais complexas dos mesmos. De forma geral, a aceitação do curso, pelos professores, e vontade de construir novas aplicações, a partir dos exemplos criados, possibilitou verificar a importância e necessidade de cursos e ferramentas como estas, que colocam o professor no controle de sua aplicação.

Nem todos os professores concederam imagens dos chatbots desenvolvidos. Mas a Figura 4 apresenta a imagem de dois chatbots exemplos criados por 2 professores durante as aulas, Rex e Celsium, o primeiro é um assistente de Petshop e o segundo um assistente de um café. 


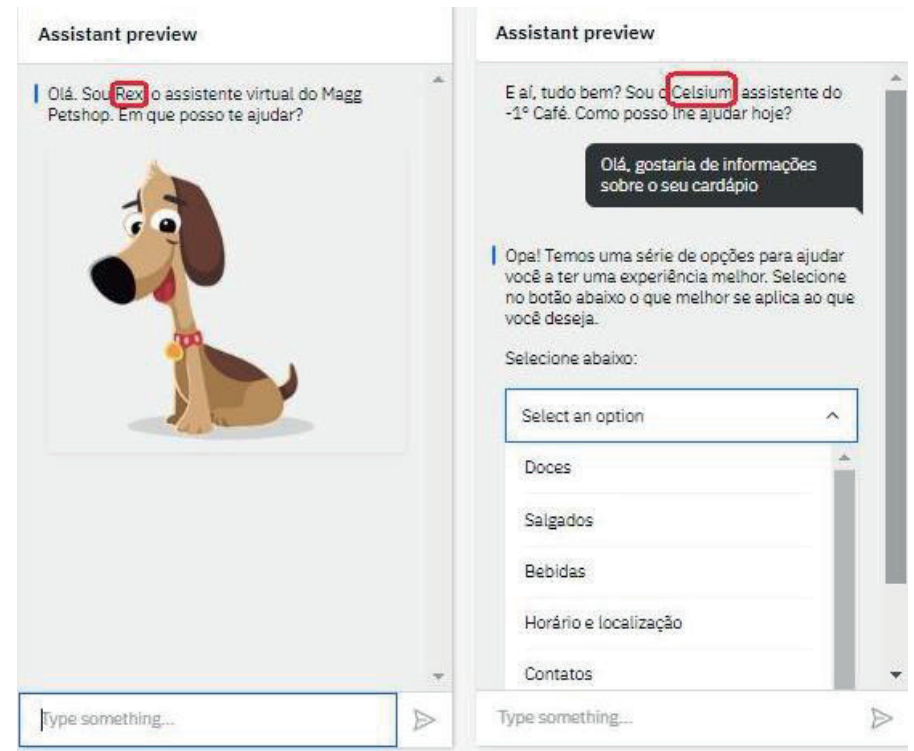

Figura 4 - Rex e Celsium, chatbots desenvolvidos por 2 alunos

Fonte: Postagem realizada por aluna em rede social

Nota-se que os professores utilizaram todos os conceitos apresentados no curso na construção de seus próprios exemplos, passos iniciais para desenvolvimento de uma ferramenta util para sua aplicabilidade em diferentes propósitos.

\section{Conclusão}

Assim como pode-se tornar o aluno protagonista do seu aprendizado sobre conteúdos em sala de aula, o professor pode se tornar protagonista do desenvolvimento de ferramentas que apoiem o ensino. Um serviço como o Watson Assistant, que tem uma interface simples e fácil de ser compreendida possibilita ao professor a possibilidade de criação de seu próprio agente conversacional como tutor em seu domínio, para auxiliar seus alunos, responder dúvidas de forma imediata, oferecer uma ferramenta motivadora e eficaz. Este trabalho, apresentou a criação de um curso introdutório de desenvolvimento de chatbot, aplicado com professores voluntários, de diversas instituições do país. Pode-se notar que utilizar o modelo de sala de aula invertida e plataformas como o Zoom e o Google Classroom contribuiu para que houvesse uma trilha de aprendizagem engajada, segmentada e motivadora. Pode-se notar que com uma capacitação de $12 \mathrm{~h} /$ aulas os professores foram capazes de desenvolverem seus primeiros chatbots, e assim, posteriormente podem aprimorarem.

A maioria dos professores do curso já haviam utilizado chatbots anteriormente como usuários para compra de produtos ou resolver dúvidas relacionadas a um determinado serviço ou sistema, mas nenhum havia desenvolvido um. Ainda, o conhecimento dos participantes sobre a aplicação aumentou de forma considerável com o curso. Houve uma resposta favorável dos professores ao curso, inclusive com a solicitação de novos módulos, que abordem conceitos mais complexos e disponibilização de novos vídeos por parte da tutora. Finalmente, esses resultados permitiram a aplicação de um curso para uma turma de professores do EJA, em andamento. 


\section{Agradecimentos}

O presente trabalho foi realizado com apoio da Coordenação de Aperfeiçoamento de Pessoal de Nível Superior - Brasil (CAPES) - Código de Financiamento 001. Agradecemos também a disponibilidade, colaboração e confiança do grupo STHEM Brasil.

\section{Referências Bibliográficas}

BARROS, D. M. V.; GUERREIRO, A.M. Novos desafios da educação a distância: programação e uso de Chatbots. Espaço Pedagógico, Passo Fundo, v. 26, n. 2, p. 410431, maio/a go. 2019.

BEIRA, D. G.; NAKAMOTO, P.T. A formação docente inicial e continuada prepara os professores para o Uso das tecnologias de informação e Comunicação (TICs) em sala de aula? In: Workshop de Informática na escola (WIE 2016) do Congresso Brasileiro de Informática na Educação. 22, Uberlandia, 2016, anais, Uberlândia: Sociedade Brasileira de Computação, 2016, p. 825-834.

DE MORAIS, A. P. M; SOUZA, P. F. Formação docente continuada: ensino hibrido e sala de aula invertida como recurso metodológico para o aprimoramento do profissional de educação. Devir Educação, ed. especial, p. 10-32, 2020, ago. 2020.

DOS SANTOS, L. F.; TEZANI, T. C. R. Aprendizagem colaborativa no ensino de história: a sala de aula invertida como metodologia ativa. RENOTE-Revista Novas Tecnologias na Educação, v. 16, n. 2, dez. 2018, p. 101-111.

HUANG, W.; HEW, K. F.; GONDA, D. E. Designing and Evaluating Three ChatbotEnhanced Activities for a Flipped Graduate Course. International Journal of Mechanical Engineering and Robotics. v. 8, n. 5, set 2019, p. 813-818.

IBM. Docs da IBM CLOUD/Watson Assistant (gerenciado). 2020. Disponível em: < https://cloud.ibm.com/docs/assistant?topic=assistant-getting-started\&locale=pt-BR $>$ Acesso em 29 out. 2020.

KUYVEN, N. L.; ANTUNES, C. A.; VANZIN, V. J. B.; SILVA, J.L.T.; KRASSMANN, A.L; TAROUCO, L. M. R. Chatbots na educação: Uma revisão sistemática da literatura. Revista Renote: Novas Tecnologias na Educação, v. 16, n.1, jul. 2018, 10p.

LEONHARDT, M. D.; NEISSE, R.; TAROUCO, L. M. R. MEARA: um chatterbot temático para uso em ambiente educacional. In: Simpósio Brasileiro de Informática na Educação, 14., 2003, Rio de Janeiro, anais, Porto Alegre: Sociedade Brasileira de Computação, 2003, p. 81-88.

MORO, F.F. Protótipo de um chatbot para auxiliar o professor na utilização do Sistema Tutor Inteligente MAZK. Araranguá: Universidade Federal de Santa Catarina, 2019, Dissertação de Mestrado.

NDUKWE, I. G.; DANIEL, Ben K.; AMADI, C. A Machine Learning Grading System Using Chatbots. Artificial Intelligente in Education: Lecture Notes in Computer Science, v. 11626, Cham:Springer, p 365-368.

YANG, S.; EVANS, C. Opportunities and Challenges in Using AI Chatbots in Higher Education. In: International Conference on Education and E-learning, 3.,2019, Barcelona, Anais, Nova York: Londres, 2019, p 79-83. 